\title{
Adaptive Ensemble Construction based on Progressive Interactive Training of Neural Networks
}

\author{
M. A. H. Akhand, Member, IACSIT and K. Murase
}

\begin{abstract}
The goal of an ensemble construction with several neural networks (NNs) is to achieve better generalization ability over a single neural network. In a Neural Network Ensemble (NNE), component networks solve the desired problem independently and combine their outputs for NNE's output. Therefore, the size of an NNE plays an important role in determining the performance for a particular problem. Although there has been much work for constructing ensembles, most of the works trains predefined number of NNs for an NNE. This study presents a problem dependent adaptive NNE construction method based on Progressive Interactive Training Scheme (PITS) of NNs. In PITS, a predefined number of NNs are trained one by one in a progressive manner, where each $\mathrm{NN}$ is concerned with a specific task that has not been solved by any previously trained NN. Proposed Adaptive PITS (APITS) is an extension of PITS utilizing inherited benefit of its sequential style training. APITS employs a simple overhead in PITS while training NNs one after another sequentially. The overhead determines more NNs training will be beneficial or not for a given problem and therefore, return different number of NNs for different problems. The proposed method has been evaluated on several benchmark problems, and the method (with concise NNE) is shown competitive generalization ability with the popular NNE methods.
\end{abstract}

Index Terms-Generalization, neural network ensemble, problem dependent adaptive ensemble.

\section{INTRODUCTION}

The goal of an ensemble construction with several Neural Networks (NNs) is to achieve better generalization ability over a single neural network. The inspiration for building an ensemble is the same as for establishing a committee of people: each member of the committee should be as competent as possible, but the members should be complementary to one another. If the members are not complementary, i.e., if they always agree, then the committee is unnecessary as any one member could perform the task of the committee. On the contrary if the members are complementary there is a high probability that the remaining members may correct the error when one or a few members make an error. Thus, for Neural Network Ensemble (NNE) construction, proper diversity among component networks is considered to be an important parameter so that the failure of one may be compensated by others.

In an NNE, component networks solve the desired problem independently and combine their outputs for NNE's output.

Manuscript received May 1, 2012; revised May 31, 2012.

M. A. H. Akhand is with the Dept. of Computer Science and Engineering, Khulna University of Engineering and Technology, Khulna-9203, Bangladesh (e-mail: akhand@cse.kuet.ac.bd).

K. Murase is Graduate School of Engineering, University of Fukui, 3-9-1 Bunkyo, Fukui 910-8507, Japan (e-mail: murase@.u-fukui.ac.jp).
Therefore, the size of an NNE plays an important role in determining the performance for a particular problem. If the size of an ensemble is smaller than necessary, the ensemble will be unable to solve problems. On the other hand, if the size is larger than necessary, the computational cost for constructing an ensemble will increase. Although there has been much work for constructing ensembles, most of the works determine an NNE size (i.e., number of networks in the NNE and architecture of individual networks) manually and maintain fix during training [1]-[6]. While manual determination of an NNE size might be appropriate for problems where rich prior knowledge and experienced experts exist; it often requires trial-and-error process for many real world problems when rich prior knowledge and experienced experts do not exist.

The problems on which Neural Network (NN) or its ensemble works belong a large variety on the basis of complexity, size, input features and output classes. Due to large variety, predefined ensemble size for all the problems might not be effective; moreover for some problem single network may be sufficient. It is empirically reported that an ensemble outperforms single network when the given problem is large and/or complex [5]. However, most of the conventional popular NNE construction methods, such as bagging [1] and AdaBoost [3] methods, always consider several component NNs to construct an NNE for a problem and the number of NNs is selected manually [1],[3],[5].

Besides training predefined number of networks, CNNE [7] builds an ensemble constructively and automatically. CNNE starts with a minimal NNE i.e., two networks with single hidden node per NN. With defined criteria, at run time it adds hidden node in the existing NNs and new network in the NNE. CNNE trains all component networks simultaneously with full training set via Negative Correlation Learning (NCL). Another point is that it starts with single hidden node per network for all the problems but some problems require more hidden nodes. Therefore, computational cost seems high due to full training set for all the networks and small number of initial hidden nodes.

For problem dependent ensemble size, DECORATE [4] uses trial and test method to select networks for an NNE. First, it trains a network with the union of diversity set (i.e., the artificially generated training patterns) and original training set. After that, performance of the NNE is checked with the trained one. If the performance degrades by the addition, DECORATE leaves this network and generates a new diversity set and trains a new network. The algorithm terminates after a desired ensemble size or a specified number of trained networks are reached [4]. DECORATE trains each individual network on relatively larger training data that is the combination of original training data and 
generated artificial data. Also it trains a large number of networks to select several networks for final ensemble. Both the factors increase the computational cost much more.

To construct minimal NNE, MNNEC [8] first tries to solve the problem with a single network, after that it builds ensemble using additional networks when single network fails to give satisfactory result. To construct ensemble with appropriate or nearly appropriate architecture, several networks are trained one after another in such a manner that sampled training data which are misclassified by previous network(s) is used to train the current network. Due to training with previously misclassified patterns, full training space is solved independently where individual networks are specialized on different portions of training space and showed high diversity among them. Finally, in order to coordinate the component networks, all the NNs in the ensemble are trained simultaneously with full training set. It uses two stage training of networks.

Regarding problem dependent adaptive NNE, this paper presents a method based on Progressive Interactive Training Scheme (PITS) [10]. The rest of the paper is organized as follows. Section II describes new adaptive NNE construction method and Section III presents experimental results. Finally, Section IV concludes the paper with brief summary.

\section{AdAPTive EnSEmble Construction USING PROGRESSIVE INTERACTIVE TRAINING OF NNS}

To make the paper self contained first we give a short description of Progressive Interactive Training Scheme (PITS) and Negative Correlation Learning (NCL) [11]. PITS is based on the theme of training time interaction of NCL and tried to resolve the difficulties of NCL. On the other hand, proposed Adaptive PITS (APITS) of this study is an extension of PITS adding a training time overhead to determine appropriate number of NNs for a problem in PITS.

The NCL trains predefined number of NNs simultaneously and interactively. NCL introduces a penalty term in the error function to establish training time interaction among NNs in the ensemble. According to [11], the error of the $i$-th $\mathrm{NN}$ in the ensemble for the $n$-th training pattern is

$$
e_{i}(n)=\frac{1}{2}\left(f_{i}(n)-d(n)\right)^{2}-\lambda\left(f_{i}(n)-f(n)\right)^{2}
$$

where $f_{i}(n)$ is the actual output of $i$-th NN; and $d(n)$ and $f(n)$ are the actual and the desired output of the ensemble for the $n$-th training pattern respectively. First portion of the equation is the back propagation error function; and second portion is the correlation penalty term that employs by NCL in which $\lambda$ is a scaling factor that controls the penalty term. For the n-th training pattern, the output of an ensemble consisting of $\mathrm{M}$ networks is

$$
f(n)=\frac{1}{M} \sum_{i=1}^{M} f_{i}(n)
$$

According to [3], the partial derivative of $e_{i}(n)$ that is require to modify the connection weights of NNs in learning process is

$$
\frac{\partial e_{i}(n)}{\partial f_{i}(n)}=f_{i}(n)-d(n)-2 \lambda\left(f_{i}(n)-f(n)\right)\left(1-\frac{1}{M}\right) .
$$

It is clear from Eq. (3) that NCL needs to know the ensemble output (i.e., $f(n)$ ) for updating the weight of each NN. This means an NN needs to communicate with all other NNs in the ensemble for updating its weight. This kind of direct interaction among all NNs in the ensemble is time consuming, and NNs may engage in competition during training [7]. In addition, the number of NNs to construct an ensemble needs to be predefined in NCL.

To reduce training time interaction and competition among NNs, PITS employs an indirect communication scheme for training NNs in an ensemble through an intermediate media called Information Center (IC). In PITS, NNs in the ensemble are trained one by one in a progressive manner, where each NN is concerned with a specific task that has not been solved by any previously trained NN. The partial derivative required to modify the weights of $i$-th NN in PITS becomes

$$
\frac{\partial e_{i}(n)}{\partial f_{i}(n)}=f_{i}(n)-d(n)-2 \lambda\left(\frac{i-1}{i} f_{i}(n)-\frac{1}{i} f_{I C}(n)\right),
$$

where $f_{I C}$ is the element from IC and is the sum of previously trained NNs.

$$
f_{\text {IC }}(n)=\sum_{j=1}^{i-1} f_{j}(n)
$$

Fig. 1 shows the graphical representation of training scheme of component networks in PITS [10]. To train a network, PITS first collects information from IC and, after training, updates IC. The idea of such indirect interaction is mimicked from ant colony system (ACO) and found effective when compared to ensemble training with direct interaction in NCL [11]. The detail description of PITS is available in [10].

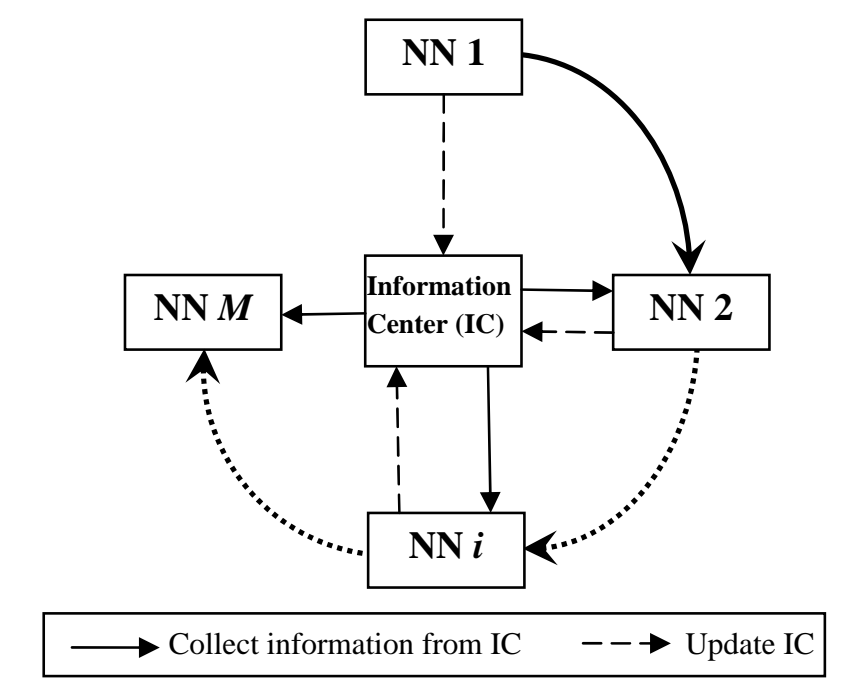

Fig. 1. Graphical representation of training scheme of NNs in PITS. To train a network, PITS first collects information from IC and, after training, updates IC [10].

The main attraction of this study is to investigate a problem dependent adaptive NNE construction method based on PITS. Proposed Adaptive PITS (APITS) is an extension of PITS utilizing inherited benefit of its sequential style training. APITS employs a simple overhead in PITS while training NNs one after another sequentially. The overhead determines more NNs training will be beneficial or not for a given 
problem and therefore, return different number of NNs for different problems.

There are several ways of anticipation whether more networks require or not for the given problem. Classification error or diversity are common for the anticipation purpose and used in DECORATE and CNNE. Besides these, overlap and uncover target space are two measures introduced recently [10]. This study uses uncover target space for this purpose. When uncover reach minimal point then stops training of more networks.

Uncover Target Space (UTS) means space where no network give correct answer. There is no chance to get better performance (i.e., classification rate) with larger UTS [9]. It is important to realize that diversity among component networks may give lower TER when failure of one network may be compensated by others. So, networks might cover full target space such a way that for each and every pattern majority number of networks might reply correct answer. Again, diversity has a strong relation with overlap because diversity among identical networks will be zero because their functions are overlapped and decisions are identical. Finally, diversity plays important role on testing error rate through uncover and overlap. The UTS is presented in this study as the rate to the total patterns and the Rate of UTS (RUTS) measured according to the following equations.

\section{$R U T S=\theta / N$}

Here $\mathrm{N}$ is the number of patterns in the training or testing set; and $\theta$ is the total number of patterns where none of the networks reply with the correct class. It is important to understand that though larger uncover never gives better TER the lowest uncover (i.e., zero or near zero) will not always give the lowest TER. The reason is that for a particular pattern if any network reply is the true class then the pattern is out of uncover; but NNE decision for the pattern comes out from the combination of all the networks' output. Finally, better TER might have the lowest uncover with a compromising value of overlap.

\section{EXPERIMENTAL STUDIES}

Several benchmark problems were considered to evaluate the performance of new adaptive NNE method. The source of the problems is the University of California, Irvine (UCI) machine learning repository [12], the most popular benchmark dataset collection for neural networks or machine learning. UCI contains raw data and requires preprocessing for use in NNs. We have followed benchmark methodology [13] for preprocessing the problems. The number of output nodes was equal to the number of classes for a given problem. Continuous input feature values were rescaled between 0 and 1 with a linear function. For discrete features, the number of inputs was selected as the number of distinct values in general. Table I shows the characteristics of problems which show considerable variety in the number of patterns, input features and classes. These problems provide a suitable experimental test bed.

In order to evaluate performance of an NNE, generalization is measured on the testing set that was reserved from available data and not seen by any $\mathrm{NN}$ in the training and selection processes. Testing Error Rate (TER) is the well acceptable measure for generalization and its value minimum is good. Notice that the aim of any NNE method is to achieve the lower TER.

TABLE I: CHARACTERISTICS OF BENCHMARK DATASETS

\begin{tabular}{|c|c|c|c|c|c|c|}
\hline \multirow{2}{*}{ Dataset } & \multirow{2}{*}{ Example } & \multirow{2}{*}{ Class } & \multicolumn{2}{|c|}{ Input Feature } & \multirow{2}{*}{$\begin{array}{l}\text { NN } \\
\text { Input }\end{array}$} & \multirow{2}{*}{$\begin{array}{l}\text { Hidden } \\
\text { Node }\end{array}$} \\
\hline & & & Cont. & Disc. & & \\
\hline Australian Credit Card & 690 & 2 & 6 & 9 & 51 & 10 \\
\hline Breast Cancer Wisconsin & 699 & 2 & 9 & - & 9 & 5 \\
\hline Diabetes & 768 & 2 & 8 & - & 8 & 5 \\
\hline House Vote & 435 & 2 & - & 16 & 16 & 5 \\
\hline Lymphography & 148 & 4 & - & 18 & 18 & 10 \\
\hline Soybean & 683 & 19 & - & 35 & 82 & 25 \\
\hline Postoperative & 90 & 3 & 1 & 7 & 19 & 5 \\
\hline Splice Junction & 3175 & 3 & - & 60 & 60 & 10 \\
\hline Wine & 178 & 3 & 13 & - & 13 & 5 \\
\hline Waveform & 5000 & 3 & 21 & - & 21 & 10 \\
\hline
\end{tabular}

It can be seen that the TER may vary due to the variation of the testing set data, even if the size of the data set remains the same. Therefore, standard 10-fold cross validations have been used for result presentation. In the cross validation, initially available training examples were partitioned into ten equal or nearly equal sets, and for each turn, one set was reserved as a testing set, while the remaining nine sets were used for training.

\section{A. Experimental Results}

This section first gives an experimental analysis on a sample problem how the measures of RUTS reflect to determine the number of NNs in the NNE for a given problem. The experiment is done on Soybean problem that is 19 class problem having 35 input features. After that, results of APITS were compared with the popular NNE methods.

Fig. 2 shows the classification error rate and uncover (i.e., RUTS) for both training and testing sets when 30 networks were trained one after another through PITS for the Soybean problem. From the figure it is clear that uncover reduces sharply for first few networks and does not improve much for the later networks. It is also found that classification error rate has a relation with uncover and it is found almost unchanged for steady uncover. Therefore, the point when uncover reaches the minimum or steady is a good choice to stop adding more networks for the NNE of the particular problem and give adaptive problem dependent NNE; this is the theme of the proposed adaptive PITS.

Table II presents the experimental results of proposed APITS and compares the results with bagging and AdaBoost, the two most popular NNE methods. The results of bagging and AdaBoost are collected from the recent study [9] in which 20 fixed numbers of networks were trained for an NNE. From the table it is clear that NNE size in APITS is adaptive with problem. For large sized or more classed problem, it constructed NNE with more networks; as an example, for Splice Junction and Waveform problems the networks in the NNE were 23.18 and 21.82, respectively. On the other hand, for Postoperative and Wine problems it built NNE with 3.46 and 4.1 networks, respectively; both the problem are three classed problem having less number of examples. At a glance, the average number of networks was 9.05 for the ten 
problems. But it is interesting to observe that the TERs of APITS with adaptive NNE size was competitive to bagging or AdaBoost with 20NNs/NNE. The average TER of APITS was 0.1254 ; on the other hand, average TERs of bagging and AdaBoost were 0.1263 and 0.1361 , respectively. Moreover, in some cases, APITS achieved the lowest TER among the three methods. As an example, TER of APITS was 0.2889 for the Postoperative problem; whereas, the TERs of bagging and AdaBoost were 0.3155 and 0.3511 , respectively. Finally, the proposed APITS is outperformed bagging and AdaBoost with concise NNE.

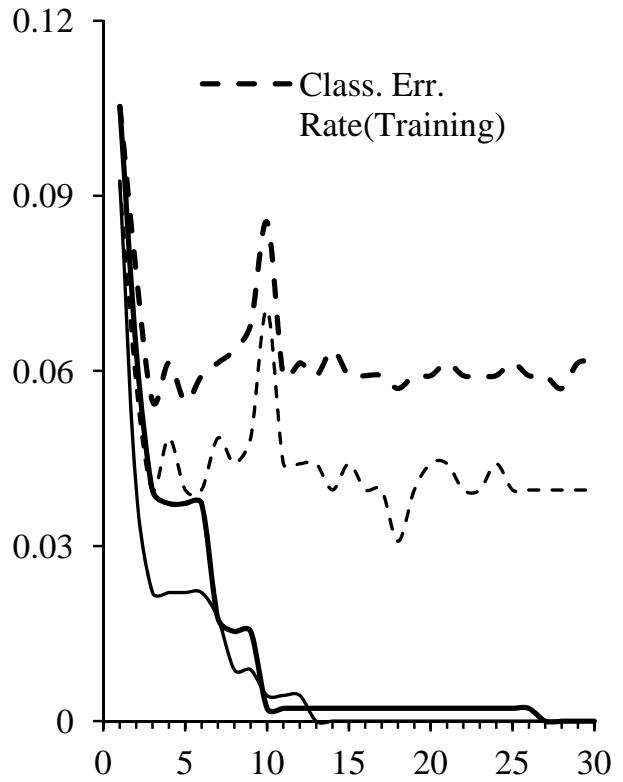

Fig. 2. Effect of NNs training in PITS for Soybean problem.

TABLE II: COMPARISON AMONG BAGGING, ADABOOST AND APITS BASED ON ACHIEVED TER AND ENSEMBLE ARCHITECTURE OVER FIVE STANDARD 10-FOLD CROSS VALIDATION RUNS.

\begin{tabular}{lccccc}
\hline \hline & \multicolumn{3}{c}{ TER } & \multicolumn{3}{c}{ NNE size (NNs/NNE) } \\
\cline { 2 - 6 } Problem & Bagging & AdaBoost & APITS & $\begin{array}{c}\text { Bagging or } \\
\text { AdaBoost }\end{array}$ & APITS \\
\hline Australian Card & 0.1417 & 0.1568 & 0.1351 & & 6.04 \\
Breast Cancer & 0.0322 & 0.0322 & 0.0313 & & 4.40 \\
Diabetes & 0.2321 & 0.2305 & 0.2347 & & 12.24 \\
House Vote & 0.0372 & 0.0437 & 0.0377 & & 5.48 \\
Lymphography & 0.1529 & 0.1729 & 0.1557 & & 3.74 \\
Soybean & 0.0517 & 0.0535 & 0.0576 & & 6.02 \\
Postoperative & 0.3155 & 0.3511 & 0.2889 & & 4.1 \\
Splice Junction & 0.1556 & 0.1529 & 0.1611 & & 23.18 \\
Wine & 0.0141 & 0.0353 & 0.0247 & & 3.46 \\
Waveform & 0.1297 & 0.132 & 0.1273 & & 21.82 \\
Average & $\mathbf{0 . 1 2 6 3}$ & $\mathbf{0 . 1 3 6 1}$ & $\mathbf{0 . 1 2 5 4}$ & $\mathbf{2 0}$ & $\mathbf{9 . 0 5}$ \\
\hline \hline
\end{tabular}

\section{CONCLUSION}

The aim of building an ensemble with several neural networks is to achieve the better performance for a given problem. Conventional ensemble methods train a predefined number of networks and all of them are involved in ensemble decision generally. This study presents a problem dependent adaptive NNE construction based on progressive interactive training of networks. The proposed method is found better than other popular traditional methods with concise ensemble when tested on benchmark problems.

\section{REFERENCES}

[1] L. Breiman, "Bagging predictors," Machine Learning, vol. 24(2), 1996, pp. 123-140.

[2] T. G. Dietterich, "Ensemble Learning," in The Handbook of Brain Theory and Neural Networks, Second edition, 2002, pp. 405-408. The MIT Press, Cambrigde, MA.

[3] Y. Freund and R. E. Schapire, "Experiments with a new boosting algorithm," Proc. of the 13th International Conference on Machine Learning, Morgan kaufmann, pp. 148-156, 1996.

[4] P. Melville, and R. J. Mooney, "Creating Diversity in Ensembles using Artificial Data," Information Fusion, vol. 6, 2005, pp. 99-111.

[5] D. W. Opitz and R. Maclin, "Popular Ensemble Methods: An Empirical Study,” Journal of Artificial Intelligence Research, vol. 11, 1999, pp. $169-198$.

[6] A. J. C. Sharkey, "On Combining Artificial Neural Nets," Connection Science, vol 8, 1996, pp. 299-314.

[7] M. M. Islam, X. Yao and K. Murase, "A Constructive Algorithm for Training Cooperative Neural Network Ensembles," IEEE Transactions on Neural Networks, vol. 14, 2003, pp. 820-834.

[8] M. A. H. Akhand, and K. Murase, "A Minimal Neural Network Ensemble Construction Method: A Constructive Approach,” Journal of Advanced Computation Intelligence and Intelligent Informatics (JACIII), vol. 11(6), pp. 582-592.

[9] M. A. H. Akhand, M. M. Islam, and K. Murase, "A Comparative Study of Data Sampling Techniques for Constructing Neural Network Ensembles,” International Journal of Neural Systems, vol. 19(2), 2009, pp. 67-89.

[10] M. A. H. Akhand, M. M. Islam, and K. Murase, "Progressive Interactive Training: A Sequential Neural Network Ensemble Learning Method," Neurocomputing, vol.73, 2009, pp. 260-273.

[11] Y. Liu \& X. Yao, "Ensemble Learning via Negative Correlation," Neural Networks, vol. 12, 1999, pp. 1399-1404.

[12] D. J. Newman, S. Hettich, C. L. Blake, and C. J. Merz, "UCI Repository of Machine Learning Databases," Dept. of Information and Computer Sciences, University of California, Irvine,1998. Available: http://www.ics.uci.edu/ mlearn/

[13] L. Prechelt, "Proben1- A Set of Benchmarks and Benching Rules for Neural Network Training Algorithms,” Tech. Rep. 21/94, Fakultat fur Informatik, University of Karlsruhe, Germany, 1994.

[14] S. Haykin, Neural Networks - A Comprehensive Foundation, Prentice Hall, 2nd edition, 1999.

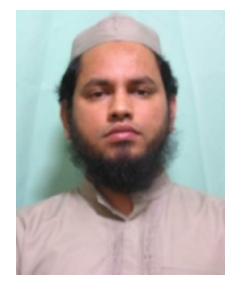

M. A. H. Akhand received the B.Sc. degree in Electrical and Electronic Engineering from Khulna University of Engineering and Technology (KUET), Bangladesh in 1999, the M.E. degree in Human and Artificial Intelligent Systems in 2006, and the Doctoral degree in System Design Engineering in 2009 from University of Fukui, Japan. He joined as a lecturer at the Department of Computer Science and Engineering at KUET in 2001, and is now an Associate Professor. He is a member of Institution of Engineers, Bangladesh (IEB). His research interest includes artificial neural networks, evolutionary computation and other bio-inspired computing techniques.

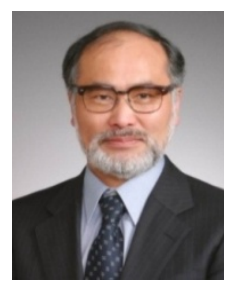

K. Murase is a Professor at the Graduate School of Engineering, University of Fukui, Fukui, Japan, since 1999. He received ME in Electrical Engineering from Nagoya University in 1978, $\mathrm{PhD}$ in Biomedical Engineering from Iowa State University in 1983. He Joined as a Research Associate at Department of Information Science of Toyohashi University of Technology in 1984, as an Associate Professor at the Department of Information Science of Fukui University in 1988, and became the professor in 1992. He is a member of The Institute of Electronics, Information and Communication Engineers (IEICE), The Japanese Society for Medical and Biological Engineering (JSMBE), The Japan Neuroscience Society (JSN), The International Neural Network Society (INNS), and The Society for Neuroscience (SFN). He serves as a Board of Directors in Japan Neural Network Society (JNNS), a Councilor of Physiological Society of Japan (PSJ) and a Councilor of Japanese Association for the Study of Pain (JASP). 